Preparedness is comprised of a response plan which is taught in basic EMS training and practiced a few times each year. The response is based on shift ambulances, Mobile Intensive Care Units (MICU), and volunteer first responders. This article proposes to study the phase which occurs after authorization by the fire department, extraction of the patients, and first triage and treatment that includes secondary triage and allocation of the patient to the appropriate transporting vehicle, with the appropriate medical team for transportation to the hospital, with consideration of injury types and severity. The departure site facilitators conduct secondary triage, ensure the proper medical team and vehicle, and report quantity of injured and severity to the receiving destination hospital.

Methods: MDA is using a departure dispatch site to make secondary triage and transportation decisions. MDA conducted a drill to compare the efficiency between the use of a dedicated app for report and decision making and the use of a smartphone messaging app that allows recording of times and voice recording.

Results: Data were extracted from both apps and compared as to time intervals, report quality, apprehension of the dispatch center, and decisions made by the dispatch center. The data were compared with consideration of data from the records of MDA representative in the receiving hospitals which records arrival of ambulances, number of injured, and injury types.

Conclusion: The messaging app allowed for quicker apprehension by the dispatch, higher quality of report, and quicker and better decisions as to the destination hospital.

Prehosp Disaster Med 2017;32(Suppl. 1):s23-s24

doi:10.1017/S1049023X17000826

\section{Pediatrics for the Non-Pediatric Provider: Kids are Just Small Adults \\ Vicki L. Sakata}

Senior Medical Advisor, Northwest Healthcare Response Network, Tukwila/WA/United States of America

Study/Objective: 1 . Provide an overview of pediatric emergency care in the US, emphasizing the fact that most children are seen in non-pediatric facilities. 2. Describe various tools available to reduce cognitive load and error when caring for children. 3. Describe the Northwest Healthcare Response Network's (NWHRN) regional Hospital Toolkit for Managing Pediatric Patients in Disaster, and the statewide trainings developed as a result.

Background: Children under the age of 18 represent approximately $25 \%$ of the total US population. Many metropolitan areas have specialized Children's Hospitals. However, studies show that the majority of pediatric ER visits are made to non-pediatric hospitals ${ }^{1}$. Therefore, pediatric specialists must continue training and engaging their non-pediatric colleagues. Initial stabilization of a child can be done by any non-pediatric emergency provider. The NWHRN has developed tools and trainings for non-pediatricians, and over the past 3 years has taught throughout Washington state.

Methods: The NWHRN is a healthcare coalition representing the 2 largest counties in Washington state. We developed a regional Hospital Pediatric Toolkit specifically for non-pediatric hospitals. ${ }^{2}$ We then created half-day workshops incorporating hands-on skills sessions. Participant evaluations are reviewed and used to improve and develop new trainings.

Results: The NWHRN Pediatric Toolkit received the 2010 NACCHO Model Practice Award (National Association of County and City Health Officials (NACCHO)). Since then eleven different hands-on pediatric training sessions have been developed. Participant evaluations have "strongly agreed" that these sessions are "valuable" and "useful learning aids". The demand for trainings continues. We have also shared these products with our colleagues in Oregon through a "Train the Trainer" Workshop. Oregon has successfully completed 2 workshops in their state.

Conclusion: Pediatric emergency care and disaster preparedness should be an everyday priority in all healthcare facilities. There are several tools available to help non-pediatric providers plan and train for the pediatric patient. Hands-on sessions have been a valuable training tool. ${ }^{1}$ Gausche-Hill, M, et al, JAMA Pediatr. 2015;169(6): 527-534. doi:10.1001/jamapediatrics.2015.138 ${ }^{2}$ www.nwhm.org/ all-documents/hospital-guidelines-for-managing-pediatricpatients-in-a-disaster/

Prehosp Disaster Med 2017;32(Suppl. 1):s24

doi:10.1017/S1049023X17000838

\section{Development and Application of an Educational Program for Medical Disaster Health Coordinators in an Earthquake and Tsunami Prone Area of Japan \\ Taichi Takeda \\ Center For Disaster Medicine, Mie University Hospital, Tsu-city, Mie/Japan}

Study/Objective: We have developed and implemented an educational program for medical professionals in an earthquake and tsunami prone area of Japan, in order to provide training on the competencies needed by medical and disaster health coordinators to run a cluster meeting.

Background: Major earthquakes with a magnitude of 8.0-9.0 are anticipated to occur on the southern coast of Japan. Most part of Mie Prefecture would likely be damaged severely by tsunami and landslides. We need to foster medical and disaster health coordinators who could serve the area's Health Emergency Management Service.

Methods: We have developed a 4-hour program for the coordinators, that includes 2-hour didactic lectures and 2-hour tabletop exercises, which will be organized by the local government. The educational contents include practical procedures necessary to function as a disaster health and medical coordinator; ie, registering and dispatching medical teams and public health teams, analyzing and assessing situations in order to plan further response to a disaster, and organizing health cluster meetings. The tabletop exercise simulates disaster response in the area where the program is conducted. It requires participants to utilize the cluster meetings to share information and dispatch each team to rescue sites, shelters and/or facilities for medical and health support. The program evaluation by the participants was anonymously conducted using a questionnaire. 